\title{
Applying Decomposition Methods to Crossword Puzzle Problems
}

\author{
Yaling Zheng \\ Constraint Systems Laboratory \\ Department of Computer Science and Engineering \\ University of Nebraska-Lincoln \\ Email: yzheng@cse.unl.edu
}

\begin{abstract}
In this paper, we investigate the performance of several existing structural decomposition methods on fully interlocked Crossword Puzzle Problems (CPPs) and draw directions for future research.
\end{abstract}

\section{Introduction}

A Constraint Satisfaction Problem (CSP) is a problem consisting a finite set of variables, each of which is associated with a finite domain, and a set of constraints over a subset of constraints. Each constraint specifies allowable tuples for a subset of all the variables. The task is to find an assignment for every variable so that all constraints are simultaneously satisfied. Although CSPs are in general in NPcomplete, it is possible and desirable to identify special properties of a problem class that can be efficiently solved.

Structural decomposition methods have been proposed for identifying tractable Constraint Satisfaction Problems (CSPs) [1-5]. The basic principle is to decompose a CSP into sub-problems that are organized in a tree structure. The subproblems are then solved independently, then the original CSP is solved in a backtrack-free manner after the tree structure is made arc-consistent, as described by Dechter and Pearl [1]. The size of the biggest sub-problem is called the width of the decomposition, and is the criterion for judging the tractability of the problem.

To the best of our knowledge, no work in the literature evaluates the performance of structural decomposition techniques on real-world problems, only Harvey and Ghose [6] and our previous work [5] conduct evaluations on randomly generated CSPs. In this paper, we study the application of structural decomposition techniques on fully interlocked Crossword Puzzle Problems. Our goal is two-fold: (1) Evaluate the performance of structural decomposition techniques on a real-world problem, and (2) Initiate the investigation of techniques for solving CPPs.

CPPs, especially those in newspapers, which are usually fully interlocked, are interesting and challenging problems for people. They receive daily wide attention, and many newspapers, such as U.S.A. Today and the New York Times, 
regularly publish CPPs. Some people make CPPs for newspapers, while some readers solve those CPPs.

We state the definitions as proposed by [7]. A crossword puzzle is defined upon an $m \times n$ grid where most, if not all, of the cells are to be filled in with characters which comprises words along horizontal and vertical axes. An open cell is a blank box destined to contain a character in the final solution of the entire puzzle. A closed cell appears as a solid box, does not contain any character and is not actually part of the puzzle but indicates an internal border. Contiguous open cells read from left to right or from top to bottom constitute words, and these contiguous cells are referred to as word slots. The degree of interlocking in a puzzle is the percentage of shared cells. A shared cell is an open cell belonging to both a vertical and a horizontal word slot. The cell in which two word slots intersect is called an orthogonal intercept. If all open cells in a puzzle are shared, the puzzle is completely interlocked. Given a word list and a grid configuration on a crossword compiler, man or machine, should find one or more solutions. A solution in this context is a filling of the grid with words all belonging to the specified word list.

A newspaper CPP is usually a fully interlocked CPP. In [8], Meehan and Gary compared two approaches modeling CPPs as CSPs: letter-by-letter and wordby-word. Also, they discussed the application of arc-consistency to the resulting problem as a pre-processing step before search. In [7], CambonJensen showed several grid-walk heuristics for finding a solution for the CPPs. However, the identification of tractable fully interlocked CPPs has not yet been studied. We are motivated to investigate whether decomposition methods is useful for solving CPPs.

In [5], we introduced four new structural decomposition methods: $\mathrm{HINGE}^{+}$, CUT, TRAVERSE, and CaT. The relationships between the decomposition methods are shown in Figure 1. The solid directed-edge from a decomposition $D_{1}$ to another one $D_{2}$ indicates that $D_{2}$ strongly generalizes $D_{1}$. The dotted directed edge from $D_{1}$ to $D_{2}$ indicates $D_{2}$ generalizes $D_{1}$. Note that the picture is incomplete in the sense that not all relationships are shown. We tested

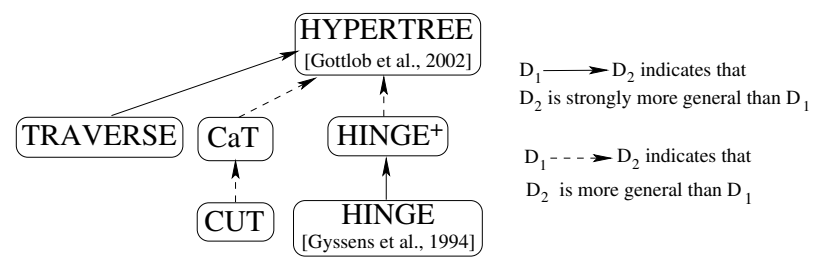

Fig. 1. Illustrating the relationships between the various studied techniques.

these methods on randomly generated CSPs. CaT, which is a hybrid of CUT and TRAVERSE, showed the best trade-off between the width of the computed constraint tree and the time for computing the decomposition In this paper, we apply these decomposition methods to fully interlocked CPPs. The experiment is done on 51 fully interlocked CPPs instances obtained from the Crossword Puzzle Grid Library [9]. 
This paper is organized as follows. Section 2 shows the decomposition result of CPPs. Section 3 discusses directions for future research.

\section{Applying structural decomposition methods to CPPs}

We apply the following decomposition methods: HINGE [3], HINGE ${ }^{+}, \mathrm{CUT}$ TRAVERSE, and CaT [5] to the associated constraint hypergraphs of 51 instances of fully interlocked CPPs obtained from Crossword Puzzle Grid Library [9]. A cut $k$ in a decomposition technique is the number of hyperedges of the constraint graph of a connected CSP that, when removed, separate the graph into two or more components. The techniques $\mathrm{HINGE}^{+}, \mathrm{CUT}$, and CaT take $k$ as a input parameter, usually starting with $k=1$, and increasing its value until the CSP can be decomposed. TRAVERSE operates by sweeping through the constraint graph, and it output depends on the starting hyperedge. Tables 1 and 2 show the CPU time for computing the decompositions and the width of those decomposition methods on the 51 instances. The $x$ axis in these figures represents the identifier of an instance. $H+1$ denotes $\mathrm{HINGE}^{+}$with $k=1, H+2$ denotes $\mathrm{HINGE}^{+}$with $k=2$, CUT1 denotes CUT with $k=1$, CUT2 denotes CUT with $k=2$, CaT1 denotes CaT with $k=1$, and CaT2 denotes CaT with $k=2$. We start TRAVERSE on each hyperedge in the problem and display the minimum value of the width as TMIN, its maximum value as TMAX, and its average value as TAVER.

Let $D$-width be the width of the join tree computed by decomposition method $D$. Note that $\mathrm{HINGE}^{+}$-width is always smaller than HINGE-width because $\mathrm{HINGE}^{+}$-width first finds cuts with size 1, then find cuts with size 2, through cuts with size $k$.

From Table 2, we notice that only for 1 out of 51 instances, the CaT-width is larger than its HINGE-width. This is instance \#27 (a $17 \times 17$ fully interlocked CPP instance ranked as difficult), as shown in Figure 2. For instance \#27, the decomposition with HINGE results into 5 large nodes shown in Figure 3. The largest node connects with the other 4 nodes via 4 different cuts in a star-shape fashion. The value of width HINGE-width for this instance is 24 . Figure 4 shows the join tree computed by CaT for instance \#27 is 27, which is larger than the one computed by HINGE. This situation arose in relatively rare cases in our experiments on random CSP and crossword puzzle problems.

Moreover, we notice that the width of the join tree computed by TRAVERSE (TMIN) is smaller than the one computed by CaT with $k=2$ in 35 out of 51 cases. This results is different from the one on randomly generated CSPs, where CaT gives the best tradeoff between the width of the computed join tree and the computation cost.

\section{Future work}

For newspaper CPPs, the decomposition result seems not particularly promising: if the candidate words for every word slot is about 1000 (when the purpose 
Table 1. CPU time of the decompositions of 51 newspaper CPPs by HINGE, HINGE ${ }^{+}$, CUT, TRAVERSE, and CaT.

\begin{tabular}{|c|c|c|c|c|c|c|c|c|c|c|c|c|}
\hline \multirow{2}{*}{$\begin{array}{c}\text { Instance } \\
\# \\
\end{array}$} & \multirow[t]{2}{*}{ ID } & \multirow[t]{2}{*}{$\# \mathrm{E}$} & \multirow[t]{2}{*}{ HINGE } & \multicolumn{2}{|c|}{ HINGE $^{+}$} & \multicolumn{2}{|c|}{\begin{tabular}{|l|} 
CUT \\
\end{tabular}} & \multicolumn{3}{|c|}{ TRAVERSE } & \multicolumn{2}{|c|}{$\mathrm{CaT}$} \\
\hline & & & & $k=1$ & $k=2$ & $k=1$ & $k=2$ & & $\max$ & \begin{tabular}{|l|l|} 
aver \\
\end{tabular} & $k=1$ & $k=2$ \\
\hline & c13-b1 & 68 & 90 & 680 & 1370 & 540 & 3500 & \begin{tabular}{|l|}
0 \\
\end{tabular} & 10 & 3.09 & 510 & 3560 \\
\hline 2 & $13-\mathrm{m} 1$ & 66 & 160 & 270 & 12760 & 270 & 12520 & 0 & & 3.03 & 280 & 12620 \\
\hline 3 & $3-\mathrm{m} 2$ & 70 & 30 & 910 & 2940 & 690 & 12520 & 0 & 0 & 3.29 & 680 & 12210 \\
\hline 4 & $3-\mathrm{d} 1$ & 60 & 30 & 510 & 2810 & 520 & 2780 & 10 & 10 & 3.33 & 530 & 2800 \\
\hline 5 & $3-\mathrm{d} 2$ & 60 & 130 & 520 & 2800 & 490 & 2780 & 0 & 0 & 3.33 & 520 & 2800 \\
\hline 6 & $5-\mathrm{b} 1$ & 90 & 290 & 1730 & 8960 & 1350 & 31850 & 10 & 10 & 5.44 & 1360 & 32030 \\
\hline & & 90 & 70 & 950 & 1140 & 910 & 1540 & 20 & & .33 & 930 & 1540 \\
\hline 8 & 5-b3 & 90 & 10 & 970 & 1060 & 920 & 1550 & 0 & 0 & 5.22 & 930 & 1550 \\
\hline 9 & $5-\mathrm{m} 1$ & 82 & 220 & 1570 & 4280 & 1230 & 9730 & 0 & 10 & 5.12 & 1220 & 9760 \\
\hline 10 & $\mathrm{c} 15-\mathrm{m} 2$ & 82 & & 1010 & 20200 & 1020 & 20110 & 10 & 0 & 5.49 & 1020 & 20250 \\
\hline 11 & c15-m3 & 80 & 340 & 550 & 44620 & 550 & 44810 & 0 & 0 & 5.38 & 530 & 45000 \\
\hline 12 & & 80 & 300 & 1310 & 10840 & 1300 & 10870 & 10 & 10 & 5.38 & 1320 & 10920 \\
\hline & & & & & 4 & & & & & & & \\
\hline 14 & & 76 & & & 42490 & 520 & 42340 & 0 & 10 & & 530 & 42700 \\
\hline 15 & $5-\mathrm{d} 3$ & 76 & 0 & 520 & 20110 & 540 & 20100 & 0 & 10 & 5.79 & 550 & 20250 \\
\hline 16 & $5-d 4$ & 74 & 290 & 500 & 34500 & 500 & 34440 & 10 & 10 & 5.40 & 510 & 34890 \\
\hline 17 & & & & 3370 & 15120 & 2700 & 26160 & 10 & 0 & 8.67 & 2720 & 26210 \\
\hline & & & & & & & & & & & & \\
\hline & & & & & 0 & 2220 & & & & & 200 & \\
\hline & & & & 80 & 5140 & 970 & 94740 & 0 & 10 & 8.10 & 990 & 95180 \\
\hline 2 & & & 0 & 00 & 63310 & 990 & 63250 & 10 & 0 & 7.8 & 1000 & 63140 \\
\hline & & & 10 & 30 & 102820 & 1010 & 102890 & 10 & 10 & 8.20 & 1030 & 103540 \\
\hline & & & & & & & & & & & 2690 & \\
\hline & & & & & & & & & & & & \\
\hline & & 96 & & & 520 & 1000 & & 10 & 1 & & 1000 & 54550 \\
\hline & & 96 & & & 67920 & 1020 & & 10 & 1 & & 1020 & 67720 \\
\hline & & & & & 1980 & 1450 & & 10 & 10 & & 1460 & 6550 \\
\hline & & & & & 3550 & 2050 & & 10 & 10 & & 2070 & 280180 \\
\hline & & & & & & & & & & & 5140 & \\
\hline & & & & & & & & & & & & \\
\hline 3 & & & & & & 1880 & & & 20 & & 1880 & 216150 \\
\hline & & & & & & & & 10 & 20 & & 1970 & 119620 \\
\hline & & & & & & 1720 & & & 10 & & 1760 & \\
\hline & & & & & & & & & & & 1850 & \\
\hline & & & & & & & & & & & & \\
\hline & & & & & & 30 & & & 20 & & 310 & \\
\hline & & & & & & & & & 20 & & 3270 & \\
\hline & & & & & & & & & 20 & & 3280 & 3548 \\
\hline & & & & & & & & & 20 & & 3040 & 259 \\
\hline & & & & & & & & & & & 329 & \\
\hline & & & & & & & & & & & 0 & \\
\hline & & & & & & & & & 2 & & 3260 & \\
\hline & & & & & & & & & 20 & & 2770 & \\
\hline & & & & & 5963 & 2210 & 454 & 10 & 10 & & 2220 & 455000 \\
\hline & & & & 64 & & 6420 & & & 10 & & 6430 & 165270 \\
\hline & & & & & & & & & & & & \\
\hline & & & & & & & & & & & & \\
\hline & & & & & & & & 10 & 10 & & 2290 & \\
\hline & & & & 2580 & & 2590 & & 10 & 10 & 12. & 2590 & 408830 \\
\hline & & & & & & 2240 & & 10 & 20 & & 2260 & \\
\hline & & 16 & 46 & 2070 & & 2060 & 334 & 10 & 10 & 11.63 & 2070 & 33442 \\
\hline
\end{tabular}

is to make a crossword or we have no clues about the word), in a decomposition of width 8 , the biggest sub-problem has a size of $10^{24}$. Thus, the required space is huge. However, since a sub-problem of fully interlocked may be highlyconnected, there may actually be few solutions to the subproblem, and it may be the realistic to find them using backtrack search with a full look-ahead technique. Therefore, for a fully interlocked CPP, if we are able to use a structural decomposition technique to identify subproblems, then find all the solutions of these subproblems, and store them without significant overhead because there number is not large, then this processing may allow us to greatly reduce the cost of solving the CPP. This would make decomposition methods practically useful.

On the other hand, we propose to investigate the use of local search for solving fully interlocked CPPs given a dictionary and a grid. Local search starts from an initial assignment, which is randomly generated, continuously improving until it 
Table 2. Width of the decompositions of 51 newspaper CPPs by HINGE, HINGE ${ }^{+}$, CUT, TRAVERSE, and CaT.

\begin{tabular}{|c|c|c|c|c|c|c|c|c|c|c|c|c|}
\hline \multirow{2}{*}{\begin{tabular}{|c|} 
Instance \\
$\#$
\end{tabular}} & \multirow[t]{2}{*}{ ID } & \multirow[t]{2}{*}{$\# \mathrm{E}$} & \multirow[t]{2}{*}{ HINGE } & \multicolumn{2}{|c|}{ HINGE $^{+}$} & \multicolumn{2}{|c|}{ CUT } & \multicolumn{3}{|c|}{\begin{tabular}{|l|} 
TRAVERSE \\
\end{tabular}} & \multicolumn{2}{|c|}{$\mathrm{CaT}$} \\
\hline & & & & $k=1$ & & $k=1$ & $k=2$ & $\min$ & $\max$ & & $k=1$ & $x=2$ \\
\hline $\begin{array}{ll}1 \\
\end{array}$ & \begin{tabular}{|l|}
$\mathrm{c} 13-\mathrm{b} 1$ \\
\end{tabular} & 68 & 36 & 36 & 19 & 52 & \begin{tabular}{|l|}
27 \\
\end{tabular} & 16 & 19 & 17.53 & 13 & 8 \\
\hline 2 & c13-m1 & 66 & 66 & 66 & 22 & 66 & 36 & 21 & 28 & 22.12 & 21 & 16 \\
\hline 3 & $\mathrm{c} 13-\mathrm{m} 2$ & 70 & 46 & 46 & 18 & 58 & 56 & 12 & 23 & $\mid 16.46$ & 21 & 22 \\
\hline 4 & $13-\mathrm{d} 1$ & 60 & 44 & 44 & 23 & 44 & 23 & 15 & 27 & 18.70 & 15 & \\
\hline 5 & $\mathrm{c} 13-\mathrm{d} 2$ & 60 & 44 & 44 & 23 & 44 & 23 & 15 & 27 & 18.70 & 15 & 9 \\
\hline 6 & $\mathrm{c} 15-\mathrm{b} 1$ & 90 & 58 & 58 & 26 & 74 & 72 & 12 & 30 & 18.53 & 19 & 20 \\
\hline & $5-\mathrm{b} 2$ & 90 & 20 & 20 & 10 & 32 & 14 & 14 & 28 & 15.87 & 10 & 10 \\
\hline 8 & $\mathrm{c} 15-\mathrm{b} 3$ & 90 & 20 & 20 & 10 & 32 & 14 & 14 & 28 & 16.56 & 10 & 10 \\
\hline 9 & c15-m1 & 82 & 50 & 50 & 50 & 66 & 66 & 19 & 36 & 24.98 & 26 & 26 \\
\hline 10 & $\mathrm{c} 15-\mathrm{m} 2$ & 82 & 75 & 75 & 31 & 75 & 31 & 17 & 35 & 25.52 & 28 & 18 \\
\hline 11 & $\mathrm{c} 15-\mathrm{m} 3$ & 80 & 80 & 80 & 66 & 80 & 66 & 17 & 32 & 23.15 & 29 & 24 \\
\hline 12 & 4 & 80 & 66 & 66 & 66 & 66 & 66 & 22 & 45 & 28.83 & 21 & 21 \\
\hline 13 & c & 78 & 7 & 78 & 50 & 78 & 62 & 18 & 36 & 22.97 & 23 & 19 \\
\hline 14 & $\mathrm{c}$ & 76 & 76 & 76 & 64 & 76 & 64 & 19 & 52 & 29.05 & 19 & 19 \\
\hline 15 & $\mathrm{c} 15-\mathrm{d} 3$ & 76 & 76 & 76 & 76 & 76 & 76 & 21 & 46 & 26.36 & 22 & 22 \\
\hline 16 & $\mathrm{c} 15-\mathrm{d} 4$ & 74 & 74 & 74 & 58 & 74 & 58 & 15 & 50 & 25.81 & 18 & 15 \\
\hline 17 & 7-b1 & 120 & 64 & 64 & 34 & 90 & 90 & 22 & 42 & 27.35 & 26 & 26 \\
\hline 18 & & & & 100 & 32 & 100 & 51 & 18 & 32 & & 23 & 13 \\
\hline 19 & $\mathrm{c} 17-\mathrm{b} 3$ & 108 & 78 & 78 & 60 & 78 & 78 & 17 & 40 & 23.00 & 19 & 19 \\
\hline 20 & $\mathrm{c} 17-\mathrm{m} 1$ & 100 & 100 & 100 & 80 & 100 & 80 & 17 & 37 & 26.54 & 17 & 25 \\
\hline 21 & $\mathrm{c} 17-\mathrm{m} 2$ & 102 & 102 & 102 & 38 & 102 & 38 & 14 & 44 & 23.27 & 14 & 14 \\
\hline 22 & & & 10 & 100 & 82 & 100 & 82 & 17 & 40 & 24.06 & 17 & 21 \\
\hline & & & & & & & 90 & & 60 & & & 20 \\
\hline 24 & $7-\mathrm{d} 1$ & 100 & 100 & 100 & 47 & 100 & 47 & 17 & 43 & 26.08 & 17 & 17 \\
\hline 25 & $\mathrm{c} 17-\mathrm{d} 2$ & 96 & 96 & 96 & 49 & 96 & 49 & 19 & 44 & 30.71 & 23 & 18 \\
\hline 26 & $7-d 3$ & 90 & 90 & 90 & 55 & 90 & 55 & 23 & 40 & 29.68 & 31 & 25 \\
\hline 27 & 4 & 76 & 24 & 24 & 24 & 50 & 50 & 31 & 52 & 41.26 & 27 & 27 \\
\hline 28 & & 136 & & 136 & 98 & 136 & 110 & 19 & 37 & & 22 & 24 \\
\hline 2 & & & & & 78 & 118 & 116 & 26 & 38 & & & 27 \\
\hline 30 & $-\mathrm{m} 1$ & 128 & & 128 & 94 & 128 & 106 & 18 & 52 & 28.25 & 26 & 21 \\
\hline 31 & - $-m 2$ & & & & 94 & 118 & 94 & 26 & 50 & & 26 & 40 \\
\hline 3 & & 12 & & & 122 & 2 & 122 & 20 & 54 & & 2 & 24 \\
\hline 33 & & 11 & & & & 116 & 108 & 18 & 54 & & 20 & 24 \\
\hline & & & & & & & & & 42 & & & 34 \\
\hline 3 & & 11 & & & & & & 21 & 55 & & 3 & 37 \\
\hline 36 & 1 & 14 & & & & 14 & 140 & 21 & 66 & & 44 & 44 \\
\hline & $c$ & & & & 138 & & 138 & 27 & 61 & & 32 & 32 \\
\hline 38 & $-\mathrm{m} 1$ & 148 & & 148 & 56 & 148 & 84 & 19 & 33 & 28.04 & 31 & 31 \\
\hline & $-\mathrm{m} 2$ & 140 & & & 6 & 140 & 65 & 25 & 54 & & 25 & 22 \\
\hline 4 & & & & & $14 \mathrm{C}$ & & 140 & 2 & 66 & & & 44 \\
\hline 41 & c2 & 13 & & & & & & 27 & 61 & & 32 & 32 \\
\hline & & & & & & & & 33 & 63 & & 34 & 34 \\
\hline & c2 2 & & & & & & & 24 & 50 & & 26 & 26 \\
\hline 44 & 3-b1 & 186 & 186 & 186 & 140 & 186 & 160 & 21 & 44 & 27.35 & 21 & 25 \\
\hline 45 & $-\mathrm{b} 2$ & 194 & & & & & 76 & 20 & 42 & 26.92 & 2 & 23 \\
\hline & & & & & & & & & 46 & & & 23 \\
\hline & & & & & & & & 27 & 62 & & 3 & 41 \\
\hline 48 & $3-\mathrm{d} 1$ & 162 & & 162 & 162 & 162 & 162 & 33 & 78 & 47. & 53 & 53 \\
\hline 49 & $3-\mathrm{d} 2$ & 184 & 184 & 184 & 128 & 184 & 128 & 25 & 68 & 39.18 & 30 & 33 \\
\hline & $3-d 3$ & 162 & 162 & 162 & 162 & 162 & 162 & 29 & 78 & 44.35 & 48 & 48 \\
\hline 51 & $\mathrm{c} 23-\mathrm{d} 4$ & 160 & 160 & 160 & 128 & 160 & 128 & 28 & 82 & $\mid 43.78$ & 28 & 29 \\
\hline
\end{tabular}

finds a legal assignment. The problem lies in finding a good heuristic suitable for improving the assignment from one step to the next. This method, as far as we know, has not yet been studied to applying for fully interlocked CPPs. Therefore, our future work includes:

1. Identifying more structural configurations of constraint graphs where some decomposition techniques yield better results than others although in general the opposite holds, and building hybrid decompositions techniques that exploit this information;

2. Tailoring existing decomposition methods for fully interlocked CPPs, so that every sub-problem, after backtrack search, has few solutions; and

3. Finding a good heuristic to applying local search for fully interlocked CPPs. 


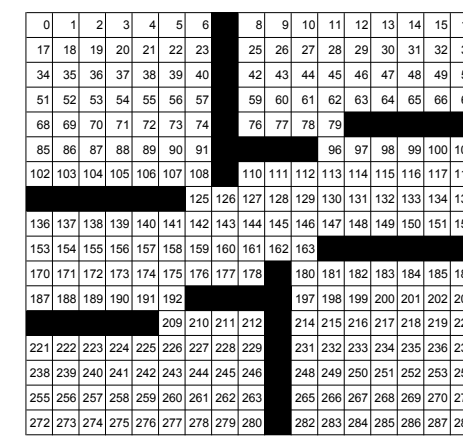

Fig. 2. Instance number \#27.
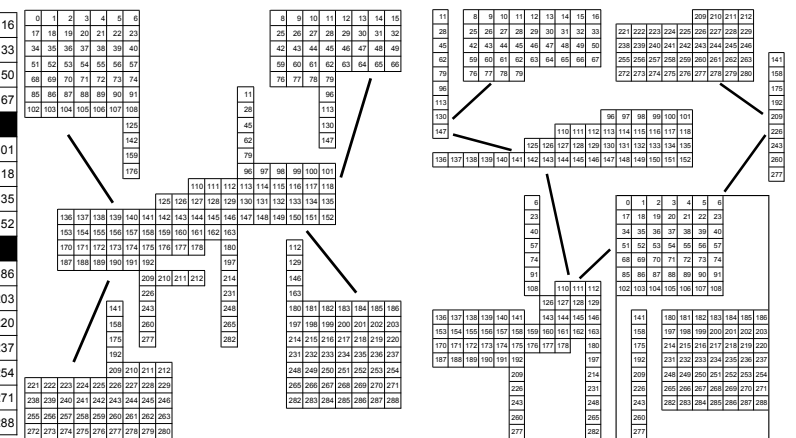

Fig. 3. Applying HINGE to Fig. 4. Applying CaT to c17-d4. c17-d4.

Acknowledgments: This work is supported by CAREER Award \#0133568 from the National Science Foundation. The experiments were conducted utilizing the Research Computing Facility of the University of Nebraska-Lincoln.

\section{References}

1. Dechter, R., Pearl, J.: Tree Clustering for Constraint Networks. Artificial Intelligence 38 (1989) 353-366

2. Gyssens, M., Jeavons, P.G., Cohen, D.A.: Decomposing Constraint Satisfaction Problems Using Database Techniques. Artificial Intelligence 66 (1994) 57-89

3. Jeavons, P.G., Cohen, D.A., Gyssens, M.: A Structural Decomposition for Hypergraphs. Contemporary Mathematics 178 (1994) 161-177

4. Gottlob, G., Leone, N., Scarcello, F.: A Comparison of Structural CSP Decomposition Methods. Artificial Intelligence 124 (2000) 243-282

5. Zheng, Y., Choueiry, B.Y.: New Structural Decomposition Techniques for Constraint Satisfaction Problems. In et al., B.F., ed.: Recent Advances in Constraints. Volume 3419 of Lecture Notes in Artificial Intelligence. Springer (2005) 113-127

6. Harvey, P., Ghose, A.: Reducing Redundancy in the Hypertree Decomposition Scheme. In: The $15^{\text {th }}$ IEEE International Conference on Tools with Artificial Intelligence (ICTAI 03). (2003) 474-481

7. CambonJensen, S.: Design and Implementation of Crossword Compilation Using Sequential Approaches Programs. Master's thesis, IMADA Odense University (1997)

8. Meehan, G., Gary, P.: Constructing Crossword Grids: Use of Heuristics vs Constraints. In: Proceedings of Expert Systems 97: Research and Development in Expert Systems XIV, SGES Publications. (1997) 159-174

9. CPPLibrary: Crossword Puzzle Grid Library. (http://puzzles.about.com/library) 